\title{
Peer review 'still essential', say researchers
}

Paris. Peer review should be adopted as the principal means of enforcing quality control in electronic science publishing, according to an international meeting of publishers, librarians, scientists and lawyers held in Paris last week. But disagreement remained about the form that such peer review should take in electronic journals.

The meeting, organized by the International Council of Scientific Unions (ISCU) and the United Nations Educational, Scientific, and Cultural Organization (Unesco), agreed that setting up secure, long-lived archives is a priority, and that common standards for indexing papers are needed to ensure accurate retrieval and cross-referencing.

It also concluded that another urgent requirement is for secure digital date stamps to tag original electronic manuscripts, as well as any subsequent modifications made to them. At present, there are no agreed rules for archiving electronic material.

The main disagreement about peer review focused on whether it should continue to be used as the sole criterion for publication of an article, or whether there is also room for electronic archives of non-peerreviewed material. The latter model has been pioneered by Paul Ginsparg, from the Los Alamos National Laboratory in California, founder of the Los Alamos e-print archives, which are now used by 40,000 physicists worldwide and which carry out 100,000 electronic transactions daily.

Ginsparg himself - who agrees that peer review is generally needed to provide quality control in the electronic literature - supports the concept of a hybrid system, with electronic archives containing submitted papers that have not been peer-reviewed coexisting with electronic journals containing papers that readers will know have been through the review process.

The advantage of such a system, he says, is that scientists would be able to choose between rapid access to non-peer-reviewed information and slower access to that filtered by peer-review, the balance between the two depending on the needs of individual disciplines.

Physicists, for example, have traditionally relied less than other disciplines on peer review, and the Los Alamos archives to a large extent simply replaced the existing tradition among physicists of distributing printed pre-prints. Indeed, Ginsparg says this, combined with the fact that the archives were quickly adopted by an active community, explains the "historical accident" of why the archives escaped the need for peer-review.

The need for electronic publishing to be tailored to the individual needs of particular disciplines was also emphasized by Sydney Hall, a crystallographer at the University of
Western Australia in Perth. Hall says he is "highly suspicious of global solutions" in that they run counter to the flexibility offered by technology.

Scientists at the meeting also criticized the fact that the peer-review process as now practised results in the publication of a single version of particular set of findings. Several scientists argued that, while peerreviewed papers could be maintained intact with a date stamp, electronic publishing offers the possibility of adding updates or related developments, each with their own time-stamp, on the original manuscript.

The meeting also identified a shift

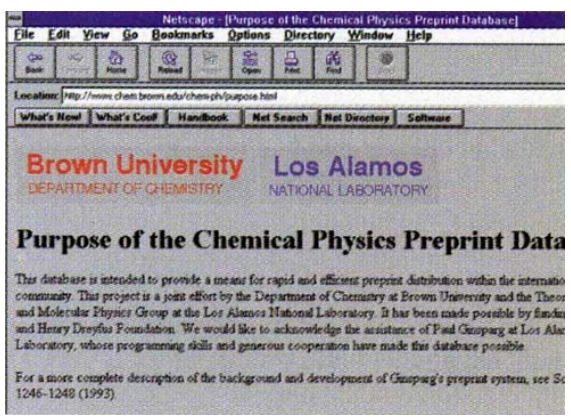

Electronic revolution: physicists have the tradition of preprints to fall back on.

towards the greater use of raw data, sounds and video in publication, and a lack of common standards for the handling of such material. For example, at least half-a-dozen incompatible protocols are now being used for rotating molecules and other figures.

The meeting stopped short of calling for new common standards to be developed. But it did recommend that "principles and guidelines" be drawn up for what is needed, reflecting the fact that science publishing is only a minor part of the electronic revolution - and that it will inevitably have to adopt standards developed by the wider multimedia industry.

Consensus was reached on many such broad issues. But deep disagreement about the role of publishers, librarians and scientists in electronic publishing remained close to the surface. Many scientists, such as Joshua Lederberg, president emeritus of Rockefeller University, New York, saw electronic publishing, for example, as a means for scientists to regain control over the distribution of scientific information from publishers primarily interested in commercial profits.

Douglas Armati, a UK-based consultant also criticized publishers for holding onto the "dogma" of a "dying transaction paradigm" in their electronic publishing ventures, based on the subscription model that has "served them well and profitably". Indeed, some scientists said they resented the large profits made by many publishing companies from their academic journal businesses, claiming that such companies often enjoyed monopolies.

Publishers have until now largely defined their operations in terms of the mechanics of distribution and production. But Armati argues that electronic publishing challenges the traditional academic publishing model of obtaining content largely without cost and then selling it back to the contributor base at high prices; publishers will face greater competition and will have to work harder to ensure that customers wish to buy their product.

Indeed, some researchers argued that their main discontent about subscription prices concerns low-circulation, high-cost journals that simply provide papers, and which they felt could be produced more cheaply electronically. High-circulation journals that offer both cheap subscriptions and additional high-quality editorial content have little to fear from the electronic revolution and everything to gain, they predicted.

But many commercial publishers and learned societies at the meeting contested the argument of Ginsparg and others that the new medium will prove much cheaper than traditional publishing operations. Major cost savings would entail researchers taking on much of the work now carried out by publishers, argued David Pullinger, electronic publisher of Nature, whereas previous experience has shown that learned societies themselves tend to set up separate publishing operations so that scientists "can get on with research".

Similarly, a representative of John Wiley \& Sons Ltd argued that the predicted increase in the electronic archival of raw data would mean that publishers would increasingly have to take responsibility for validating such data, and that this would bring new costs.

Indeed, the general mood of the meeting was that databases validated according to sets of established rules would play an increasing role in scientific communication, with a consequent shift in emphasis from peer review, which is used mainly to assess an author's interpretation of data, to a wider concept of quality control.

Programmes such as the Hubble Space Telescope (HST) and the Human Genome Project will provide useful precedents for validation procedures, argued some, pointing out that astronomers accept, for example, that data from the HST that has been validated by the Space Science Telescope Institute "corresponds to what is happening up there". Others pointed out that such validation procedures will also be important in archiving the vast amounts of declassified information emerging from defence programmes and drug companies which is too copious to be peer-reviewed.

Declan Butler 